\title{
Experimental investigation of the Multipoint Ultrasonic Flowmeter
}

\author{
Filipský Jakub ${ }^{1}$ \\ ${ }^{1}$ CTU in Prague, Faculty of Mechanical Engineering, Department of Fluid Mechanics and Thermodynamics, Technická 4, 166 \\ 07 Praha 6, Czech Republic
}

\begin{abstract}
The Multipoint Ultrasonic Flowmeter is a vector tomographic device capable of reconstructing all three components of velocity field based solely on boundary ultrasonic measurements. Computer simulations have shown the feasibility of such a device and have been published previously. This paper describes an experimental investigation of achievable accuracy of such a method. Doubled acoustic tripoles used to obtain information of the solenoidal part of vector field show extremely short differences between the Time Of Flights (TOFs) of individual sensors and are therefore sensitive to parasitic effects of TOF measurements. Sampling at $40 \mathrm{MHz}$ and correlation method is used to measure the TOF.
\end{abstract}

\section{Introduction}

The Multipoint Ultrasonic Flowmeter introduced previously in [1] is an implementation of a vector tomographic method. Tomographic methods have been widely used in several fields such as medical imaging, non-destructive testing, or oceanography. The general concept resides in the reconstruction of interior of media based on boundary measurements of some signal, which passes through that media and is influenced by its properties. This means that tomographic methods are highly non-invasive (that is the reason why their driving field is medical imaging). Also we generally receive information about properties of medium at a much higher spatial resolution compared to conventional single-point measurement methods, although point-wise precision of tomographic methods is somewhat lower.

Vector tomography specifically aims at the reconstruction of a vector quantity inside a volume, Norton [2] was the first one to introduce the necessary mathematical apparatus needed to reconstruct the vector field. When the vector quantity is fluid velocity, then ultrasound is the signal of choice.

The analytical solution to reconstruction problem given by [2] uses the Radon transform and needs a complete data set of parallel measurements taken from all possible angles. This is rather impractical for general implementation and soon many alternative reconstruction solutions began to emerge. A nice overview and division of possible approaches into groups is given by Jovanovic [3]. The solution in [1] is a $l_{2}$ norm based regularized solution that is additionally regularized by the equation of continuity.

Norton [2] realized, as well as some others later on, that only the irrotational vector field can be reconstructed from the longitudinal interaction with the measurement signal (i.e. time-of-flight), while both longitudinal and transversal interactions are needed to reconstruct the solenoidal component of the vector field [4]. When talking about fluid velocity fields and ultrasonic waves, then the transversal interaction influences the angle-of-departure and angle-of-arrival at the transmitter/receiver side respectively [3]. While the longitudinal interaction is well understood and easily described (and generally used in sonic anemometers, e.g. [5]) the transversal interaction has not been given much interest by the researchers and it is of interest to experimentally investigate the accuracy that can be achieved. Two different approaches to a practical realization of such a measurement were suggested by Jovanovic [3] - the Blumlein microphone and the acoustic tripole. The acoustic tripole method is verified experimentally in this paper as it is not very well examined in literature and still remains one of the uncertainties of the Multipoint Ultrasonic Flowmeter.

\section{Transversal flow interaction measurement}

Each vector field can be decomposed into its solenoidal (divergence free) and irrotational (curl free) components, we call this the Helmholtz decomposition. It was first shown by Norton [2] and later by others that only irrotational part of vector fields can be reconstructed from longitudal interaction (time-of-flight) measurement, while both longitudinal and transversal interaction are needed to reconstruct the solenoidal part [4]. The transversal interaction can be obtained from ultrasonic measurement also but besides simple time-of- 
flight between transceiver pairs there also needs to be an information of the angle-of-arrival (AoA) or angle-ofdeparture from the transmitter or receiver respectively.

The more straight forward method of AoA measurement is the use of the Blumlein pair which is widely used in stereo recording. It consists of two matched receivers (microphones, ultrasonic receivers) with a known directional sensitivity, positioned at different angles. Jovanovic [3], [6] proposes a different technique called the acoustic tripole. An acoustic tripole consists of three nodes - either three transmitters for angle-of-departure measurement, or three receivers for AoA measurement. Such an acoustic tripole consisting of receivers $\mathrm{A}, \mathrm{B}$, and $\mathrm{C}$ is sketched in Figure 1. Let us suppose that the receiver is sufficiently far away from the transmitter and we can neglect the curvature of the ray path. The ray arrives at the tripole traveling at group velocity $\boldsymbol{u}_{g}$ which is the vector sum of local flow velocity and the wavefront velocity. The fact that the wavefront is not traveling at its normal direction is important since the difference between TOF sensed by receivers $\mathrm{A}, \mathrm{B}$, and $\mathrm{C}$ is not dependent on the wavefront normal but rather on the group velocity.

The original acoustic tripole introduced by Jovanovic [3] is different in the sense that the receiver $\mathrm{C}$ on Figure
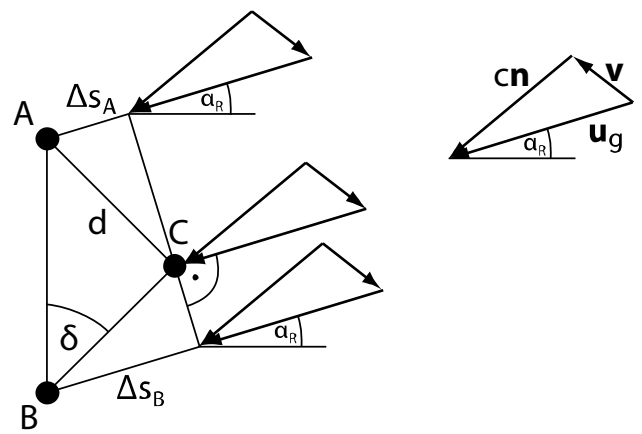

1 would be to the left of receivers $\mathrm{A}$ and $\mathrm{B}$. The choice Fig. 1. Acoustic tripole

of different layout is solely for the purpose of easier practical implementation. Also Jovanovic uses the absolute TOF sensed by the receivers for AoA computation. The author of this paper finds that rather impractical since the precise detection of ultrasonic signal arrival is complicated with physical receivers implementation. A differential TOF measurement is implemented for reasons stated later in this paper, resulting in the calculation of TOF difference with respect to some reference measurement. Let us suppose that the reference measurement will be sampled with surrounding fluid at rest. Further let $\tau_{A}, \tau_{B}, \tau_{C}$ be the TOF sensed by receivers $\mathrm{A}, \mathrm{B}$ and $\mathrm{C}$ respectively; $\tau_{A}^{0}, \tau_{B}^{0}, \tau_{C}^{0}$ are TOF sensed at rest, $\Delta s_{A}, \Delta s_{B}$ are differences of path lenghts that the ray needs to reach the receivers; rest of the notation is apparent from Figure 1. It is only a matter of geometrical constraints resulting from the sketch in Figure 1 that the difference between absolute TOF values sensed by receivers $\mathrm{A}$ and $\mathrm{C}$ is:

$$
\tau_{C}-\tau_{A}=\frac{d}{u_{g}} \sin (\delta-\Theta)
$$

For the case of reference measurement the group velocity in equation 1 will be replaced by speed of sound when the fluid is at rest :

$$
\tau_{C}^{0}-\tau_{A}^{0}=\frac{d}{c_{0}} \sin (\delta-\Theta)
$$

Combining equations 1 and 2 and expressing the same for receiver pair $\mathrm{B}$ and $\mathrm{C}$ we obtain a set of nonlinear equations for two unknowns $\Theta$ and $u_{g}$ :

$$
\begin{aligned}
& \sin (\delta-\Theta)-v\left(\frac{\Delta \tau_{A}}{d}+\sin \frac{\delta}{c_{0}}\right)=0 \\
& \sin (\delta+\Theta)-v\left(\frac{\Delta \tau_{B}}{d}+\sin \frac{\delta}{c_{0}}\right)=0
\end{aligned}
$$

The system needs to be solved numerically. Once we know the group velocity $\boldsymbol{u}_{g}$ we further need the knowledge of local fluid velocity to be subtracted from $\boldsymbol{u}_{g}$ in order to arrive at the angle $\Theta_{R}$. Jovanovic suggests to use the acoustic tripole as a standard sonic anemometer to measure the local fluid velocity. It is important to note that the work of Jovanovic concerns tomographic reconstruction of wind speeds in the atmosphere and so the scale of the acoustic tripole may be as large as practical constrains allow it to be. This means that fluid can freely flow between the receivers or transmitters. However in our case the scale of the acoustic tripole is of concern since the size of the experiment is a lot smaller. It is also important to note that in the case when the receivers or transmitters of the acoustic tripole are not submerged in the fluid flow (e.g. local fluid velocity is zero), then we only need receivers $\mathrm{A}$ and $\mathrm{B}$ as seen in Figure 1 (acoustic dipole). This can be advantageous when receivers/transmitters can be positioned outside of the fluid flow (e.g. further in the wind tunnel test section).

\section{Ray tracing}

The general task of ray tracing is to find such a path of ultrasonic waves propagating through media which satisfies the shortest time-of-arrival at its destination. This topic of acoustics geometry is well covered by several authors in [7], [8], [9], [10]. The original solution by Uginčius [8], [11] is implemented in this paper, only a brief description is presented here and the reader is referred to available literature for full mathematical derivation. The solution is based on the eikonal equation well known from optics geometry, for the case of acoustic rays in a moving, inhomogenous medium the eikonal equation takes the form

$$
|\nabla \boldsymbol{\Phi}|=\frac{c_{0}}{c+\boldsymbol{v} \cdot \widehat{n}} \equiv \mu,
$$

where $\widehat{\boldsymbol{n}}=\boldsymbol{\nabla} \boldsymbol{\Phi} / \mu$ is the unit vector in the direction normal to the wavefront. Further quantities are introduced to simplify the relations

$$
N \equiv(\mu / c) u_{g}, \boldsymbol{V} \equiv(\mu / c) \boldsymbol{v}
$$

where $\boldsymbol{v}$ represents local fluid velocity and $u_{g}$ is the magnitude of local group velocity. The eikonal equation then takes the form 


$$
\nabla \Phi=N r^{\prime}-V
$$

where $\boldsymbol{r}$ is the position vector of ray path expressed in global coordinate system and $\boldsymbol{r}^{\prime}$ is its corresponding derivative with respect to the arc length. Equation (7) is then differentiated with respect to the arc length:

$$
\left(N \boldsymbol{r}^{\prime}\right)^{\prime}+\boldsymbol{r}^{\prime} \times(\nabla \times \boldsymbol{V})=\nabla N
$$

Equation (8) represents a set of three ordinary second-order differential equations in $\mathbb{R}^{3}$ space. The system needs to be integrated numerically with initial conditions for $\boldsymbol{r}$ (position of the transmitter) and $\boldsymbol{r}^{\prime}$ (initial ray angle). If ray angle measurements are taken at the receiver side, then initial ray angle at the transmitter side needs to be iterated in order for the ray path to hit the receiver. It is of interest that equation (8) does not contain the term $\boldsymbol{V}$ in its zeroth derivative and so it is only the curl of local velocity $\boldsymbol{v}$ that matters. There are two important practical impacts on AoA measurement and fluid transversal interaction in general:

- The ray path will always be a straight line in a homogenous velocity field.

- Let us consider a case of homogenous velocity field with both transmitting and receiving site being submerged in the fluid in such a layout that the homogenous flow velocity is perpendicular to the line defined by transmitter and receiver. The ultrasonic ray travelling as a straight line will arrive at the receiver with zero AOA and thus will carry no information about the velocity field.

\section{Ultrasonic TOF measurement}

Ultrasonic TOF measurement has become a standard part of multiple industries ranging from sonic anemometers, medical imaging, non-destructive testing, to car parking sensors. The driving industry in this field has been medical imaging, much useful information is found in [12] and [13].

The requirement for precision of TOF measurement is directly connected with the AoA sensing principle. If we suppose two ultrasonic receivers spaced by $36 \mathrm{~mm}$ (that is the case of the experimental verification introduced later), then for $5^{\circ}$ of incidence angle the difference between TOF sensed by the two receivers will be in the order of $10 \mu \mathrm{s}$ when operating in air at normal temperatures. There are several ways of detecting the beginning of the ultrasonic signal, ranging from simple thresholding, through envelope fitting, to the brute-force of correlation. Comparison of four selected methods is given in [14] with the expected result of correlation being the most precise method with the highest cost and computational burden. This will be the method of choice for the experimental investigation introduced later in this paper.

Operational frequency of ultrasonic apparatus is often chosen high since higher frequencies offer higher bandwidth and higher spatial (and temporal) resolution of the signal. As shown by Cheeke [12], the directivity of the transceivers lowers down (e.g. narrower beam) with higher frequencies for constant dimensions of the transceiver. The ultrasonic vector tomograph, as suggested in the introduction to this paper, requires the transceivers to be as wide-angle as possible to get a valid signal between as many ultrasonic pairs as possible. This requirement comes handy since there is a wide range of inexpensive $40 \mathrm{kHz}$ transceivers available.

\section{Experimental verification}

A simple experiment was carried out in order to obtain validation data for the above apparatus. A setup consisting of a single transmitter and 3 receivers forming an acoustic tripole was prepared. Inexpensive off-theshelf $40 \mathrm{kHz}$ transmitter/receivers were used, signals at the electrical port of the receivers were sampled by an 8-channel (only 3 channels were connected) synchronous $40 \mathrm{MHz}$ A/D converter. The ultrasonic setup was positioned at the exit of a wind tunnel nozzle with a homogenous (piston) flow. There were three configurations of transmitter/receiver positions with respect to the flow: both

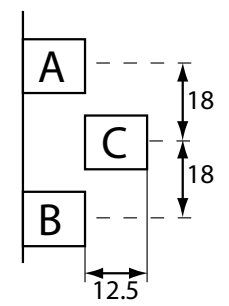

Fig. 2. Dimensions of the acoustic tripole ends well outside of the flow (Figure 3.A); transmitter at the border of the flow (Figure 3.B) and both ends at the border (Figure 3.C). No experiment with ultrasonic transmitter/receivers submerged in the flow was done since the ray path would take a straight line connecting the transmitter and receiver as noted previously.

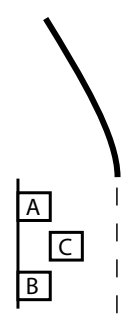

A.

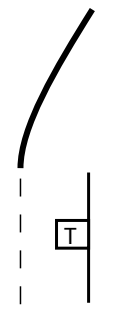

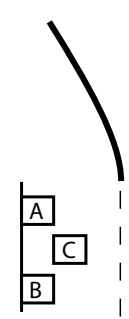

B.

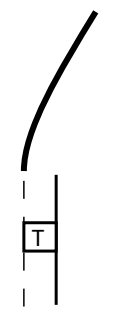

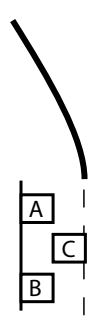

C.

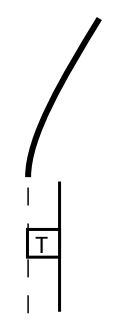

Fig. 3. Three geometrical configurations of the experimental setup 
The dimensions of the acoustic tripole are sketched in Figure 2. The width of the flow (ray path length intersecting the flow) was $950 \mathrm{~mm}$, when the nodes were positioned outside of the flow their distance to the flow stream was $120 \mathrm{~mm}$. Each measurement was done for 0, $5,10,20$, and $30 \mathrm{~m} / \mathrm{s}, 3$ samples per each prescribed velocity.

AOA dependence on velocity is plotted for each measurement setup A, B, and $\mathrm{C}$ in Figure 4. It is apparent that highest sensitivity was achieved with setup $\mathrm{C}$ where the ray path intersecting fluid with zero velocity was shortest. Lowest sensitivity is observed in setup A.

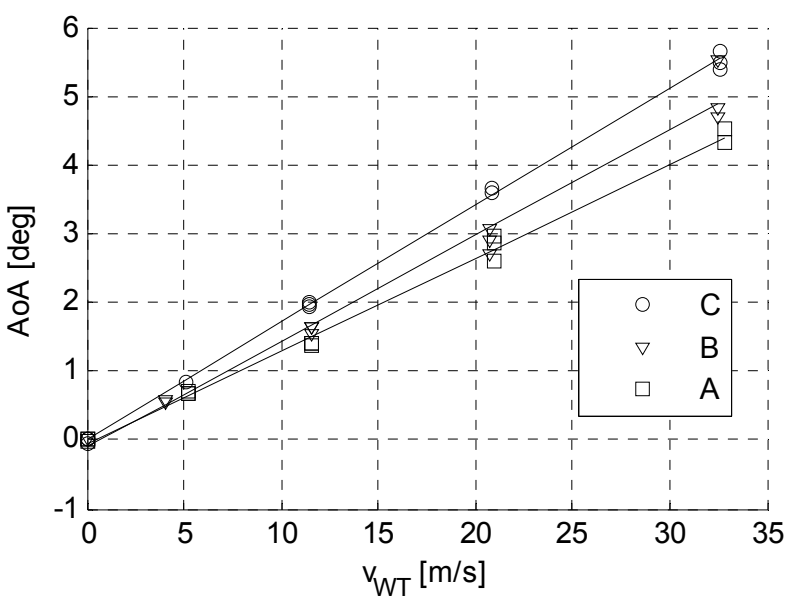

Fig. 4. Measurdd AoA dependence on velocity

Let us now convert the AoA to velocity domain. Of course there is not enough information to reconstruct the velocity distribution along the ray path, not to mention that only the rotational part of the flow field is being seen by the AoA measurement alone. Instead a normalized velocity field was prescribed with the value of one where the ray path crosses the fluid stream and zero elsewhere. Ray tracing algorithm was then run on this etalon flow field, iteratively scaling it so that the ray arrives at the acoustic tripole at the same AoA as evaluated from the experiment. The whole iterative scheme was a cascade of two iteration loops:

- Inner loop iterates the angle of departure at the transmitter side so that the ray hits the receiver

- Outer loop iterates the scaling of the etalon flow field until the desired AoA was reached

The result of the iteration is a simplified velocity profile along the ray path, which, to the author's best knowledge, is a valid assumption of the flow conditions near the exit of the wind tunnel nozzle. The velocity of the simplified profile can be directly compared to the velocity indicated by the wind tunnel. Percentage of error is plotted in Figure 5 such that positive error indicates that velocity computed from AoA is higher than velocity measured by the wind tunnel.
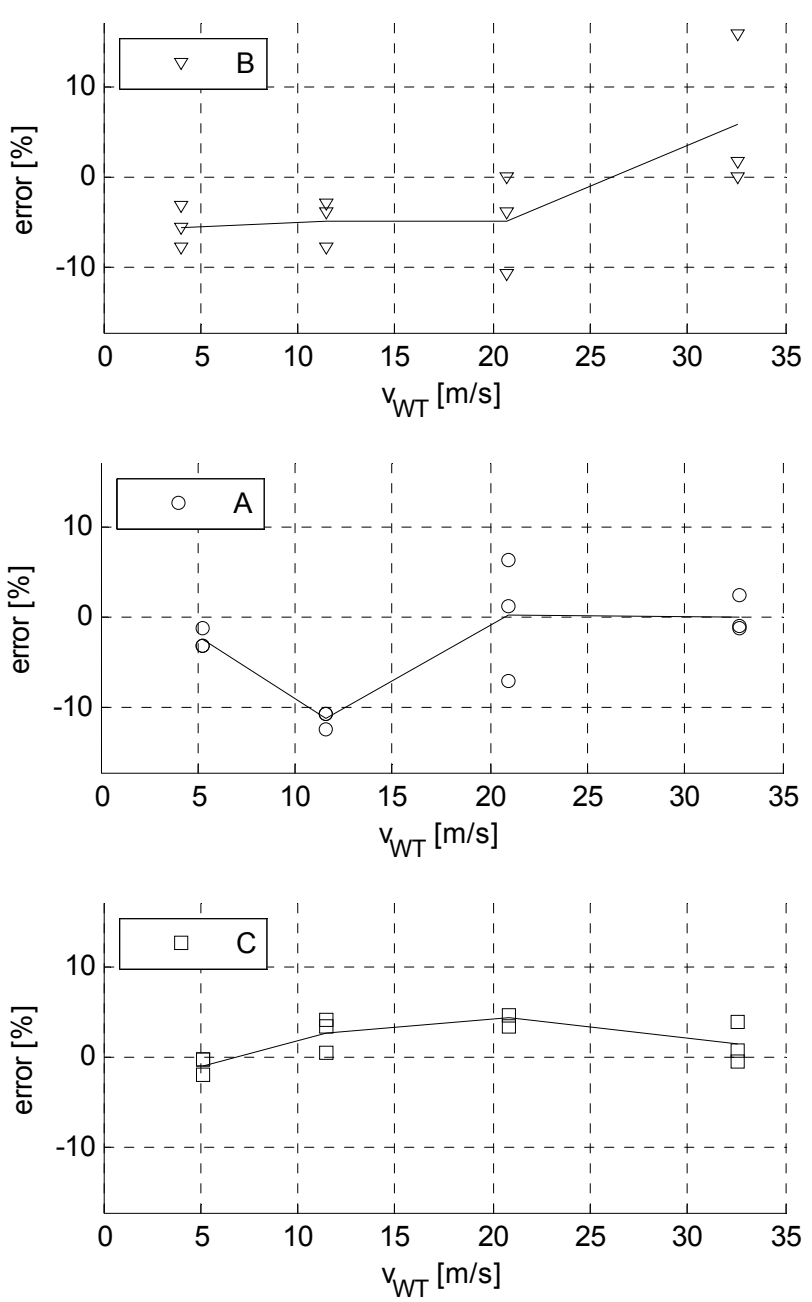

Fig. 5. Velocity error of simulation compared to the experiment

\section{Conclusion}

A simple yet robust experimental setup was proposed and carried out to test the accuracy achieved by the acoustic tripole at three geometric configurations with respect to the flow. The receiving side was positioned outside of the flow, thus surrounded by fluid at rest similar test results might have been obtained by a simpler acoustic dipole although that was not the scope of this paper. Sensitivity of AoA to velocity change was lower as there was more fluid along the ray path with zero velocity; this is in good agreement with the ray tracing simulation. The error of evaluated velocity is higher for the cases with lower sensitivity (also the noise seems a lot higher) but the large increase in performance for geometrical case $\mathrm{C}$ can hardly be explained simply by sensitivity rise. The fluid outside the flow was probably not at full rest as there are vortices forming near the stream exiting the nozzle. These vortices give a good reason for the worsened performance of cases A and $\mathrm{B}$. If we consider case $\mathrm{C}$ as the only valid experiment then the resulting accuracy is well within the range of $\pm 5 \%$. The method chosen for TOF detection $40 \mathrm{MHz}$ sampling and subsequent correlation - leaves very little doubts on the side of signal processing and 
most of the error will be caused by geometrical uncertainties on the side of the acoustic tripole and also on the side of the velocity field exiting wind tunnel nozzle. Any small deviations of AoA measurement caused by misalignment of the acoustic tripole were mostly eliminated by the use of reference reading at zero velocity. This would be valid exactly if the AoA on TOF difference (sensed by the receivers of the acoustic tripole) dependence was linear, which is true for very small misalignment angles. Further improvements in accuracy could be achieved by identification of coordinates of the receivers with respect to each other, rather than measuring them physically. This could be done by several reference readings from different angles with fluid at rest.

\section{References}

1. Filipský, J.; Nožička, J., (2016): Simulation of Multipoint Ultrasonic Flowmeter, In: STČ 2016. Praha: ČVUT, FS Ú-12242, VCSVTT, 2016. pp. 81-86. ISBN 978-80-01-05929-6.

2. Norton, S.J., (1988): Tomographic reconstruction of 2-D vector fields: application for flow imaging, In: Geophysical Journal, 97:161-168

3. Jovanović, I., L. Sbaiz, and M. Vetterli, (2009): Acoustic Tomography for Scalar and Vector Fields: Theory and Application to Temperature and Wind Estimation, In: J. Atmos. Oceanic Technol., 26, 1475-1492, https://doi.org/10.1175/2009JTECHA1266.1

4. Braun, H., Axel Hauck, (1991): Tomographic reconstruction of vector fields. IEEE Trans. Signal Processing 39: 464-471.
5. A. Coppin, P \& J. Taylor, K., (1983): A three component sonic anemometer/thermometer system for general micromeleorology research. BoundaryLayer $\quad$ Meteorology. 27. 27-42. 10.1007/BF00119970.

6. Jovanovic, I., Sbaiz, L., Vetterli, M., (2006): Acoustic Tomography Method for Measuring Temperature and Wind Velocity. IV - IV. 10.1109/ICASSP.2006.1661175.

7. Landau, L. D. ; Lifshitz, E. M., (1959): Sykes, J. B.; Reid, W. H.: Fluid Mechanics : Pergamon Press Oxford, England

8. Ugincius, P., (1971): Acoustic Rays in a Moving Inhomogeneous Medium. Journal of The Acoustical Society of America - J ACOUST SOC AMER. 49. . 10.1121/1.1975587

9. J. Thompson, Robert. (1972). Ray Theory for an Inhomogeneous Moving Medium. The Journal of the Acoustical Society of America. 51. 1675. 10.1121/1.1913014.

10. Ostashev, V. E., (1997): Acoustics in Moving Inhomogeneous Media. E\&FN SPON, 259 pp.

11. Munk, W, Worcester P, Wunsch C. (1995): Ocean acoustic tomography. , Cambridge, England: Cambridge University Press

12. J. David N. Cheeke, (2017): Fundamentals and Applications of Ultrasonic Waves, Second Edition, March 29, 2017 by CRC Press Reference - 504 Pages - $161 \mathrm{~B} / \mathrm{W}$ Illustrations ISBN 9781138077201 - CAT\# K34283

13. Szabo, Thomas L.: Diagnostic Ultrasound Imaging Inside Out A volume in Biomedical Engineering, ISBN: 978-0-12-680145-3

14. Billur, B. (2000): Fast processing techniques for accurate ultrasonic range. 0957-023 